\title{
Tracking the phase-transition energy in disassembly of hot nuclei
}

\author{
C. B. Das ${ }^{1}$, S. Das Gupta ${ }^{1}$, L. Beaulieu ${ }^{2}$, T. Lefort $^{3}{ }^{*}$, K. Kwiatkowski ${ }^{3} \dagger$, V. E. Viola ${ }^{3}$, S. J. Yennello ${ }^{4}$, L. \\ Pienkowski $^{5}$, R. G. Korteling ${ }^{6}$, and H. Breuer ${ }^{7}$ \\ 1 Physics Department, McGill University, Montréal, Canada H3A 2T8, \\ ${ }^{2}$ Department de Physique, Université Laval, Quebec, Canada G1K $7 \mathrm{P}_{4}$ \\ ${ }^{3}$ Department of Chemistry and IUCF, Indiana University, Bloomington, Indiana 47405 \\ ${ }^{4}$ Department of Chemistry and Cyclotron Laboratory, Texas A \& M University, College Station, Texas 77843 \\ ${ }^{5}$ Heavy Ion Laboratory, Warsaw University, 02-093 Warsaw, Poland \\ ${ }^{6}$ Department of Chemistry, Simon Fraser University, Burnaby, British Columbia, Canada V5A $1 S 6$ \\ ${ }^{7}$ Department of Physics, University of Maryland, College Park, Maryland 20742
}

(November 4, 2018)

\begin{abstract}
In efforts to determine phase transitions in the disintegration of highly excited heavy nuclei, a popular practice is to parametrise the yields of isotopes as a function of temperature in the form $Y(z)=z^{-\tau} f\left(z^{\sigma}\left(T-T_{0}\right)\right)$, where $Y(z)$ 's are the measured yields and $\tau, \sigma$ and $T_{0}$ are fitted to the yields. Here $T_{0}$ would be interpreted as the phase transition temperature. For finite systems such as those obtained in nuclear collisions, this parametrisation is only approximate and hence allows for extraction of $T_{0}$ in more than one way. In this work we look in detail at how values of $T_{0}$ differ, depending on methods of extraction. It should be mentioned that for finite systems, this approximate parametrisation works not only at the critical point, but also for first order phase transitions (at least in some models). Thus the approximate fit is no guarantee that one is seeing a critical phenomenon.

A different but more conventional search for the nuclear phase transition would look for a maximum in the specific heat as a function of temperature $T_{2}$. In this case $T_{2}$ is interpreted as the phase transition temperature. Ideally $T_{0}$ and $T_{2}$ would coincide. We invesigate this possibility, both in theory and from the ISiS data, performing both canonical $(T)$ and microcanonical $\left(e=E^{*} / A\right)$ calculations.

Although more than one value of $T_{0}$ can be extracted from the approximate parmetrisation, the work here points to the best value from among the choices. Several interesting results, seen in theoretical calculations, are borne out in experiment.
\end{abstract}

25.70.-z, 25.75.Ld, 25.10.Lx

\section{INTRODUCTION}

In studies of phase transitions in the disintegration of highly excited heavy nuclei, a popular path for deducing the occurrence of a phase transition is to examine the yields of composites. These are readily available from experimental data and hence have been the focus of many theoretical studies [1]. The usual practice is [2] to use a parametrisation

$$
Y(z)=z^{-\tau} f\left(z^{\sigma}\left(T-T_{0}\right)\right)
$$

and extract values of $\tau, \sigma$ and $T_{0}$, which occur in models of critical phenomena [2]. The parameters $\tau$ and $\sigma$ are critical exponents and $T_{0}$ is the critical temperature. Alternately, in a microcanonical formalism one would write

$$
Y(z)=z^{-\tau} f\left(z^{\sigma}\left(e-e_{0}\right)\right)
$$

Here $e=E^{*} / A$, the excitation energy per nucleon, and $e_{0}$ would be the phase transition energy. Formulae (1.1) and (1.2) assume that the thermodynamic limit is reached. In practice, in the nuclear case we have a finite system that disintegrates and thus the above parametrisation is only approximate. Hence the values of the parameters can be extracted in more than one way and these values may not be the same. We point out that it is largely the Coulomb force that leads different methods to give two different values for $e_{0}$, labeled as $e_{1}$ and $e_{1}^{\prime}$.

\footnotetext{
${ }^{*}$ Present address: Laboratoire de Physique Corpusculaire de Caen, F-14050 Caen Cedex, France

${ }^{\dagger}$ Present address: Los Alamos National Laboratory, Los Alamos, NM 87545
} 
Alternative but perhaps more common tools for studies of phase transition in other fields of physics are measurements of compressibility, specific heat etc. Experimental data for specific heat were studied in the nuclear case and was indeed the cause of great excitement [3].

We have therefore two distinct ways of trying to deduce a phase transition energy: from the distribution of composites as the excitation energy is varied (as explained, even here there can be more than one value) or, what may be more difficult but achievable, to locate an extremum of the specific heat. We label the excitation energy at which the specific heat maximises as $e_{2}$. It is not obvious that the values of $e_{1}, e_{1}^{\prime}$ and $e_{2}$ are close, although from the seminal work of Coniglio-Klein [4] on clusterisation this result could be anticipated.

We have compared both approaches in the nuclear case in the framework of two models. Although the models are very different and each has its own strengths and weaknesses, both reveal the following interesting features. If we switch off the Coulomb interaction between protons, the deduced phase transition energies, $e_{1}, e_{1}^{\prime}$ and $e_{2}$ are close. With the inclusion of the Coulomb force, $e_{1}$ and $e_{1}^{\prime}$ begin to diverge. For a nucleus of the size of ${ }^{197} \mathrm{Au}$, the case we study and for which fragmentation data exist, the difference in the $e_{1}$ and $e_{1}^{\prime}$ is significant. Further, one of these values stays close to the value at which the specific heat maximises and gives a good measure of the phase transition energy.

The two models we use are the Lattice Gas Model (LGM) [1,5] and a thermodynamic model [6,7]. The second model is close in spirit to the Statistical Multifragmentation Model of Copenhagen [8]. We choose to use a microcanonical simulation for LGM. So here the primary quantity is the excitation energy $e$ per particle and a temperature can be derived afterwards [9]. For the thermodynamic model, we do a canonical calculation so that the temperature is the primary parameter and an excitation energy $e$ per particle can be derived afterwards.

In section 2 we give details of the LGM calculations. Results of the thermodynamic model are presented in section 3. In section 4 we investigate the ISiS data within those formalisms. We present summary and conclusion in section 5 .

\section{RESULTS FROM LGM}

Numerical techniques for microcanonical simulations with LGM have been published [9]. Calculations are done for fixed $E=A e$ where $e$ is the excitation energy per nucleon. This is the primary quantity for simulations. The temperature for each simulation can be calculated from $T=<2 E_{k i n}>/ 3$. This is discussed in detail in [9]. For more discussions about the LGM with Coulomb force we refer to [10], section II. Bonds due to nuclear forces are taken to be $-5.33 \mathrm{MeV}$ between unlike particles and 0 between like particles. The LGM has several drawbacks, the most noticeable being the lack of quantum effects, which leads to an incorrect caloric curve near $T=0$. The LGM has the following advantages not shared by several other models. It includes interactions between composites. It incorporates the Coulomb interaction in a much more basic fashion (at the nucleonic level) than several other models. This is very important for us since this work points to a new effect brought about solely by the Coulomb interaction. Also the LGM produces particle-stable composites [10,11] so that the complicated problem of subsequent particle evaporation is circumvented.

All calculations reported here are for $Z=79$ and $N=118\left({ }^{197} \mathrm{Au}\right)$. At each total energy we compute averages after 50,000 simulated events. We use $9^{3}\left(\rho / \rho_{0}=.27\right)$ lattice sites.

The extraction of parameters from yields (Eq.(1.2)) merits consideration. Discussions of this parametrisation can be found in [2] where it is used to model a continuous phase transition in an infinite system. As already stated, one does not expect the above parametrisation to be exact except in the thermodynamic limit. For very finite systems as is the case with disintegrating nuclei formed in very energetic nuclear collisions, the parametrisation is only approximate and is by no means a signature of a critical phenomenon but rather that of a phase transition in a finite system, first order or otherwise [12]. Theory demands that $z$ of the yields be not small. In the nuclear case it is also not too big since the disassembling system itself is very finite. We limit $z$ between 3 and 17 , which is similar to most published work on the subject. Since the fit is expected to be only approximate, there is more than one prescription for getting the "best" parameters. Here we follow the prescription given in 13:

(1) If Eq. (1.2) were exact, then at $e=e_{0}$ we would have $\sum(\ln Y(z)-\ln C+\tau \ln z)^{2}=0$, as each individual term in the sum would be zero. Of course Eq.(1.2) is not exact and thus the sum above will not be zero at any value of $e$. However, this is a valid question to ask. At any given $e$ how well does the distribution fit a power law and what is the value of $\tau$ that gives a best fit to a power law ? At each $e$ we get a "best" $\tau$ by a least-square fit, i.e., by minimising $\sum(\ln Y(z)-\ln C+\tau \ln z)^{2}$ with respect to $\tau$ and $C$. The "goodness" of fit is given by the smallness of the sum which we define to be $\chi^{2}$ (there are other definitions of $\chi^{2}$ )

$$
\chi^{2} \equiv \frac{1}{N} \sum(\ln Y(z)-\ln C+\ln z)^{2}
$$


Here $N$ is the number of terms in the sum. From this step we have a "best" $\tau$ and a $\chi^{2}$ vs. e. One obvious choice of $e_{0}$ is that value of $e$ where $\chi^{2}$ is minimum (see [14]). The value of $e_{0}$ deduced using this criterion will be called $e_{1}^{\prime}$. While this is quite reasonable, it does not use the scaling property $z^{\sigma}\left(e-e_{0}\right)$ at this stage at all. That is left upon an optimum choice of $\sigma$ afterwards. The more complicated procedure that is followed below is designed to give better scaling properties.

The "best" $\tau$ vs. e curve will usually have a minimum which we call $\tau_{\min }$.

(2) Define $q=z^{\sigma}\left(e-e_{0}\right) ; f(q)$ has a maximum for some value of $q=\tilde{q}$ : $f_{\max }=f(\tilde{q})$. For each $z$ the yield $Y(z)$ as a function of $e$ has a maximum at some value of $e_{\max }(z)$. At this excitation energy $Y(z)_{\max }=z^{-\tau} f_{\max }$ where $f_{\max }$ is a constant independent of $z$. This allows us to choose values for $\tau$ and $f_{\max }$ using a $\chi^{2}$ test.

(3) The value of $\tau$ found above will be higher than $\tau_{\min }$. This means if we look for $e$ appropriate for $\tau$, two values are available from the $\tau$ vs. $e$ curve (see Fig. 1). The lower value is chosen as the value of $e_{0}$. The scaling property is badly violated by the other choice. The value of $e_{0}$ chosen by this prescription will be labelled by $e_{1}$.

(4) Now that we know $e_{0}=e_{1}$ and $e_{\max }(z)$, the excitation at which each $z$ is maximised, we find form a least squares fit the value of $\sigma$ from the condition $z^{\sigma}\left(e_{\max }-e_{1}\right)=$ const. for all $z$.

(5) The scaling law can now be tested by plotting $Y(z) z^{\tau}$ vs. $z^{\sigma}\left(e-e_{1}\right)$. Plots for all $z$ should fall on the same graph.

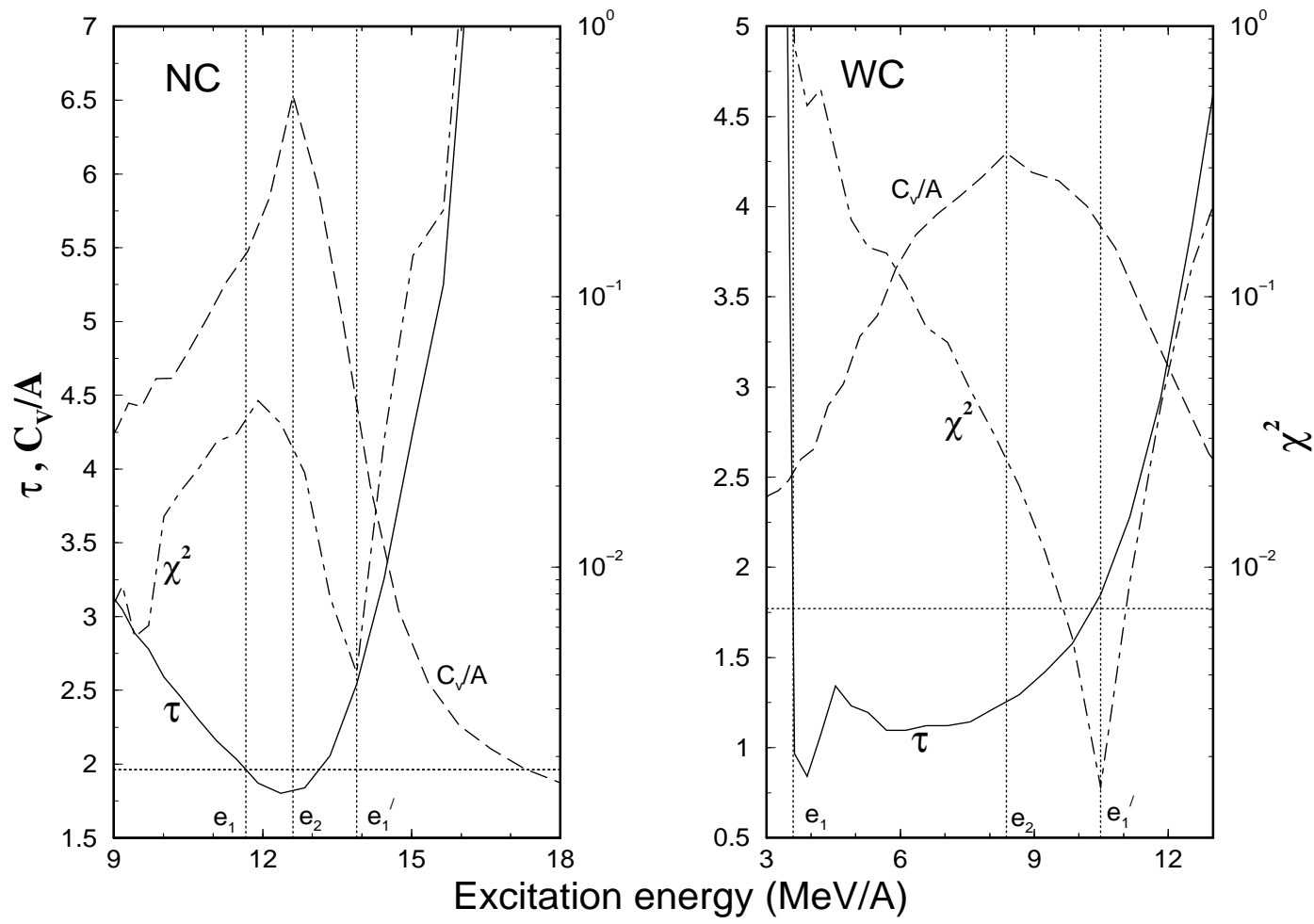

Fig.1: In this figure we show "best" $\tau$ and $\chi^{2}$ vs. e (step (1), section II) and also $C_{V}$ vs. e for the LGM calculation. The horizontal line below 2 is a straight line drawn at the value of $\tau$ from step (2), section II. The locations $e_{1}, e_{2}$ and $e_{1}^{\prime}$ are the values of $e$ for the scaling fit, maximum of specific heat and the minimum of $\chi^{2}$. The left panel is an LGM calculation without any Coulomb force, the right panel is with the inclusion of Coulomb. Notice the increase of $\Delta e=e_{2}-e_{1}$ when Coulomb is included.

Fig.1 depicts graphs (steps (1) to (3)) with and without the inclusion of the Coulomb force. In the graphs we also plot the specific heat per nucleon. We refer to the location of the maximum of specific heat as $e_{2}$. The lessons from LGM that we like to emphasize can be learned from Figs. (1) and (2). In Fig. 1 consider first the no-Coulomb case. Here $e_{1}$ is $11.66 \mathrm{MeV}, e_{2}$ is $12.61 \mathrm{MeV}$, and $e_{1}^{\prime}$ (defined by the minimum of $\chi^{2}$, the prescription of [14], see part (1) above) is $13.88 \mathrm{MeV}$. We regard them as close. With Coulomb $e_{1}$ drops well below $e_{2}$ and $e_{1}^{\prime} ; e_{2}$ and $e_{1}^{\prime}$ stay close.

Of the three energies $e_{1}, e_{2}$ and $e_{1}^{\prime}$, which one marks phase transition better? Without Coulomb, the $Y(z)$ curves at the three $e$-values are quite similar, but with Coulomb, $e_{1}$ is clearly in the phase co-existence region and is below the phase transition energy (please see Fig. 2). Looking at yields at $e_{2}$ and $e_{1}^{\prime}$, there is no obvious choice in deciding which marks the phase transition point better. However, since an extremum in the value of specific heat is a standard 
signature of phase transition, our preference is with $e_{2}$.

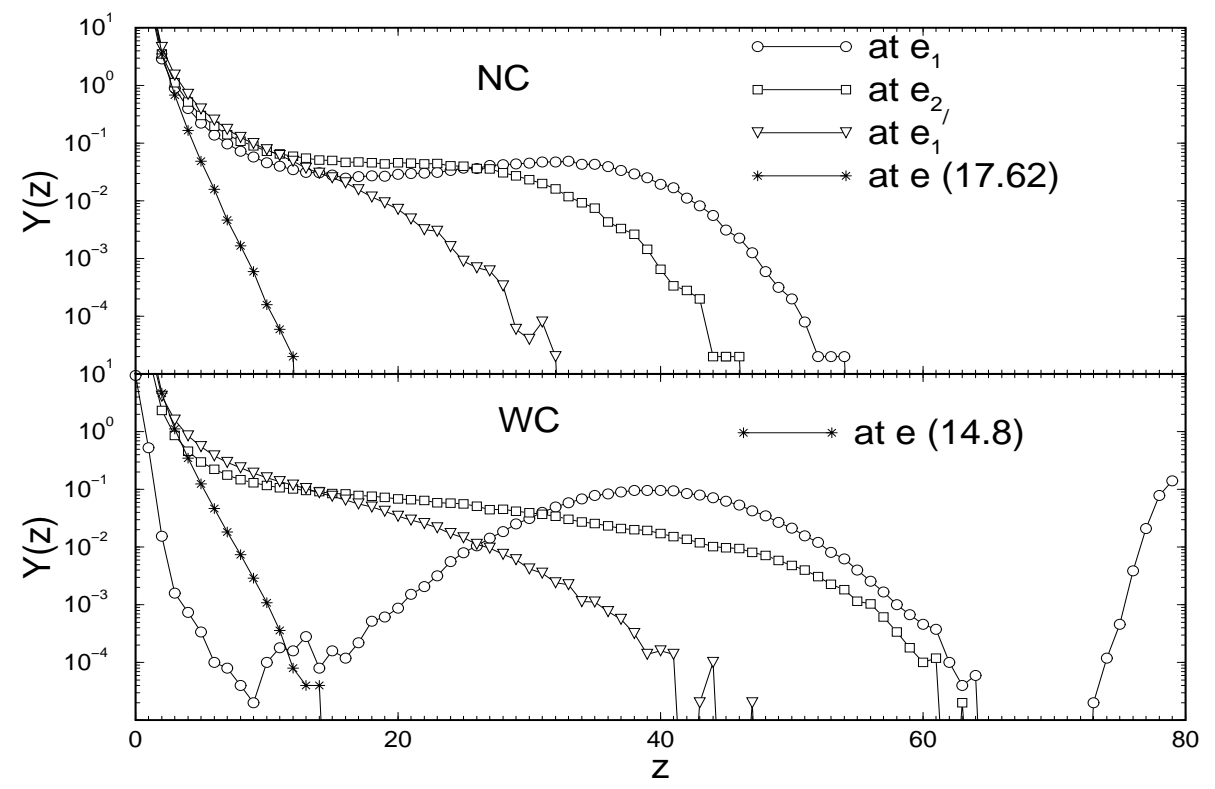

Fig.2: LGM simulations for $Y(z)$ vs. $z$ at $e_{1}, e_{2}$ and $e_{1}^{\prime}$ without Coulomb (top panel) and with Coulomb (bottom panel). In the top panel both $e_{1}$ and $e_{2}$ are near the energy, where a maximum in the yield at the high $z$ side has just disappeared. Qualitatively, this marks the phase transition point. But in the bottom panel where the Coulomb force is included, $e_{1}$ marks an energy when there is still a large fragment. Thus this is below the phase transition temperature. However $e_{2}$ still marks the location when the maximum at the high z side has just disappeared. At much larger $e$ values (shown arbitrarily at $e=17.6 \mathrm{MeV}$ and $e=14.8 \mathrm{MeV}$ ), $Y(z)$ falls much more rapidly with $z$.
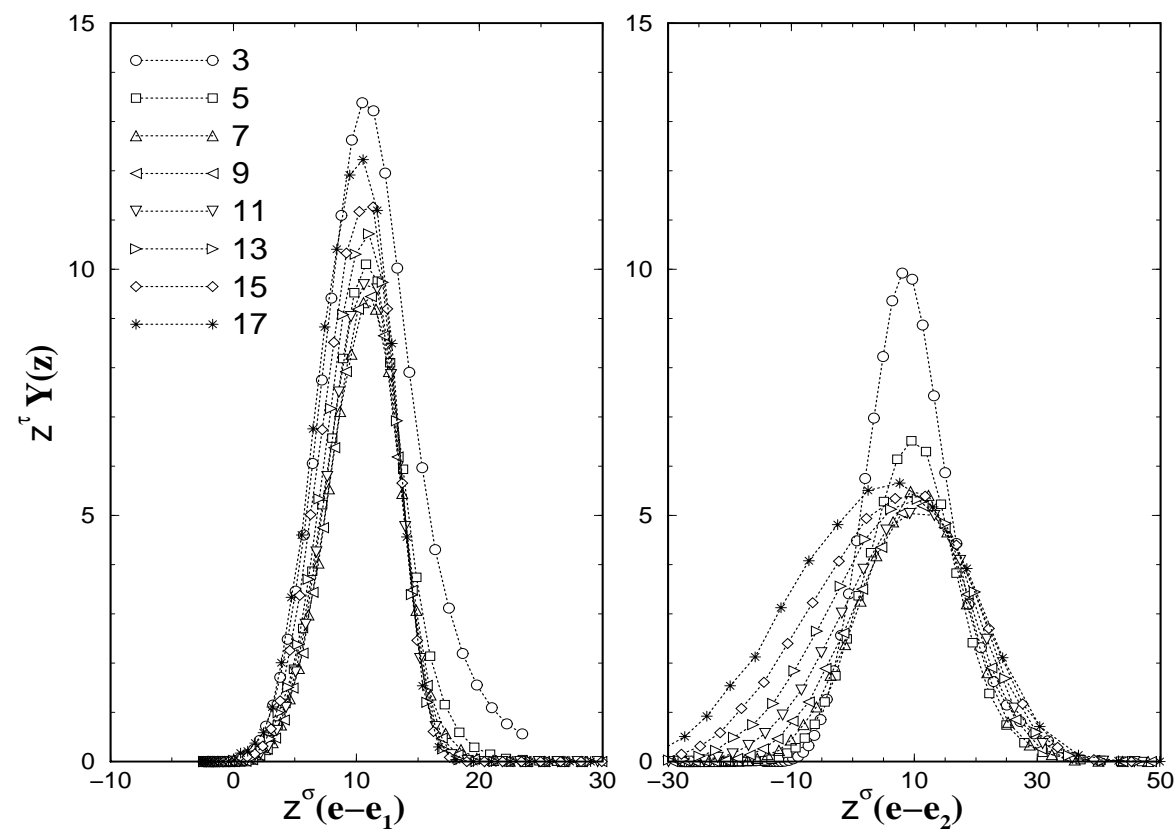

Fig.3: For different isotopes z we plot $z^{\tau} Y(z)$ against $z^{\sigma}\left(e-e_{1}\right)$ in the LGM, where the exponent $\tau$ is the "best" $\tau$ at $e_{1}$ and extraction of $\sigma, e_{1}$ is described in the text. By scaling one means that curves for different $z$ 's coalesce into one. This is approximately true for scaling around $e_{1}$ (left panel) but not around $e_{2}$ (right panel). Scaling around $e_{1}^{\prime}$ is worse (not shown). 
In Fig. 3 we show that the scaling law is rather well obeyed around $e_{1}$. It is very poorly obeyed around $e_{1}^{\prime}$. An interesting plot is a scaling law around $e_{2}$. This is also shown in Fig. 3. Of course the scaling around $e_{2}$ is nowhere as good as around $e_{1}$ but it is still better than around $e_{1}^{\prime}$ (not plotted).

\section{CALCULATIONS WITH A THERMODYNAMIC MODEL}

Details of the thermodynamic model can be found in several places [1, 6, ,7]. The physics assumption is that composites are formed at an appropriate temperature at a volume larger than normal nuclear volume dictated solely by consideration of phase space. Thus the model is close in spirit to the Statistical Multifragmentation Model of Copenhagen [8] with the simplification that the freeze-out volume is assumed to be independent of the partitions. This allows for very quick computation without any Monte-Carlo simulations. The inputs for this calculation are the following. Apart from neutrons and protons, experimental binding energies and ground state spins are used for deuteron, triton, ${ }^{3} \mathrm{He}$ and ${ }^{4} \mathrm{He}$. No excited states are included for these. For mass 5 and higher we use the semi-empirical formula for binding energies with volume term, surface tension term, symmetry energy and Coulomb energy. Excited states for composites are included in the Fermi-gas approximation. The Coulomb interaction between different composites is included in the Wigner-Seitz approximation [8].

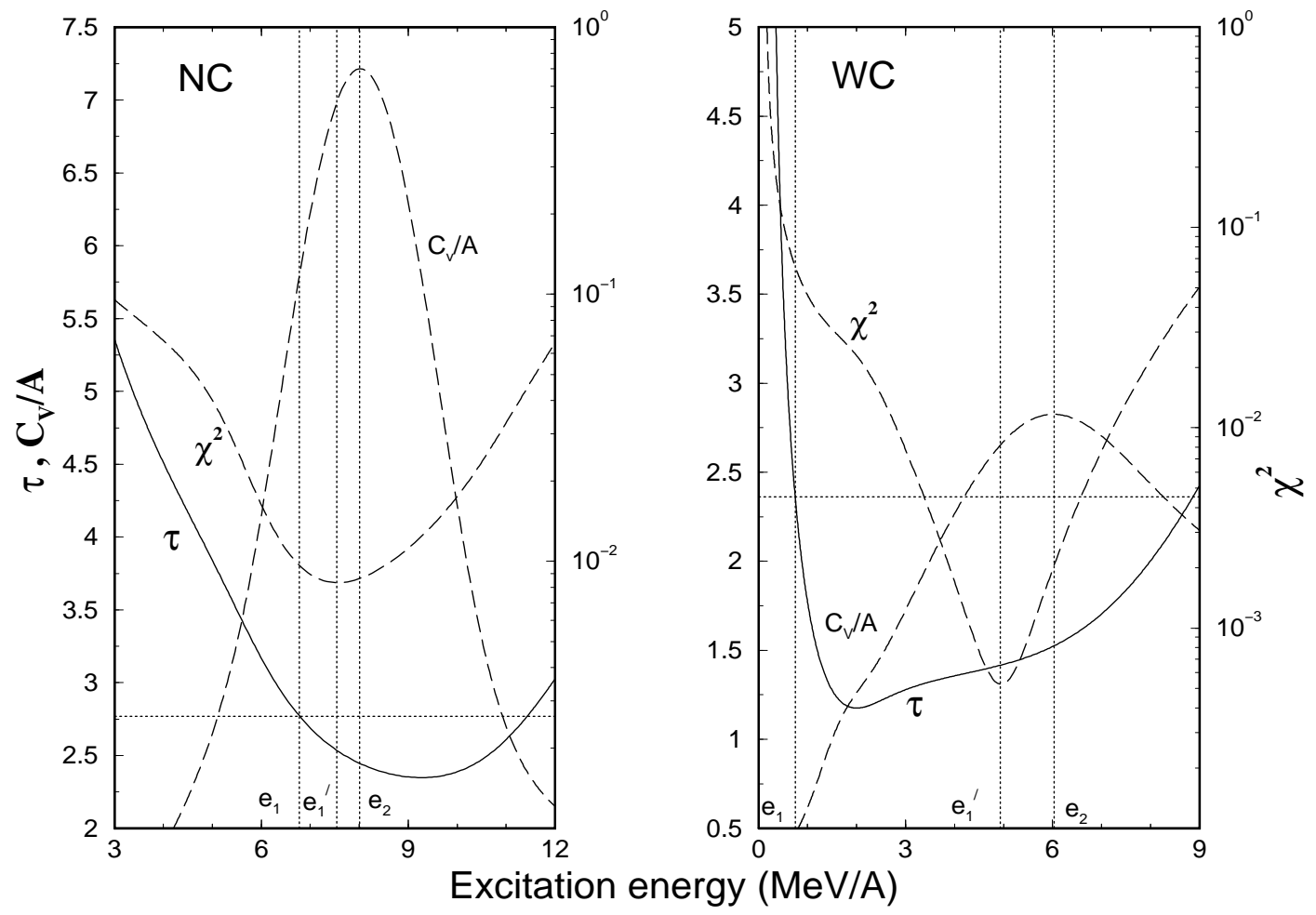

Fig.4: Similar to Fig.1 except the calculation is with the thermodynamic model (section III), with a freeze-out density $0.27 \rho_{0}$. Again note that with the inclusion of the Coulomb force (WC), $\Delta e=e_{2}-e_{1}$ increases substantially compared to no Coulomb (NC) case.

Since this is a canonical calculation, calculations are done for fixed temperatures. For each temperature, the average excitation energy per particle can be calculated. For comparisons with LGM, figures are drawn with energy as the abscissa. Table I gives both the temperature and energy for relevant quantities.

There is no reason to expect results close to the ones calculated using LGM. For example, $e_{1}^{\prime}$ is lower than $e_{2}$ in the thermodynamic model but higher in the LGM. Nonetheless, Fig. 4 shows that $\Delta e=e_{2}-e_{1}$ increases significantly with inclusion of the Coulomb force (from $1.24 \mathrm{MeV}$ to $5.28 \mathrm{MeV}$ ). Again $e_{1}$ does not seem to mark the point of phase transition at all (Fig. 5) and $e_{2}$ is a much better candidate. This is so in spite of the fact scaling is well obeyed with respect to $e_{1}$ and not so well with respect to $e_{2}$ or $e_{1}^{\prime}$ (Fig. 6). 


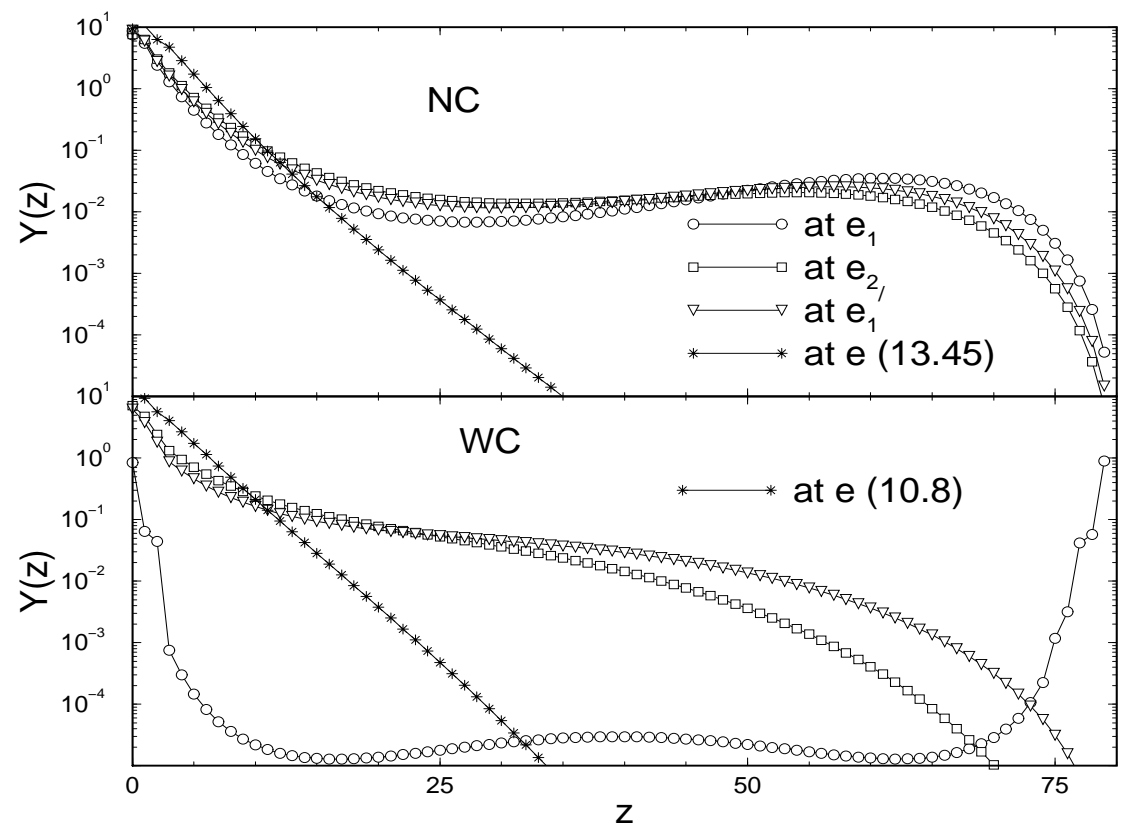

Fig.5: Similar to Fig. 2 except the calculation is with the thermodynamic model (section III). Again with (WC) and without $(N C)$ the Coulomb interaction, $e_{2}$ continues to be a better mark for the point of phase transition energy. Without the Coulomb both $e_{1}$ and $e_{2}$ are acceptable. At higher energies (chosen arbitrarily at e=10.8 MeV and 13.45 $\mathrm{MeV}$ ) the drops of yields with $z$ are much faster.
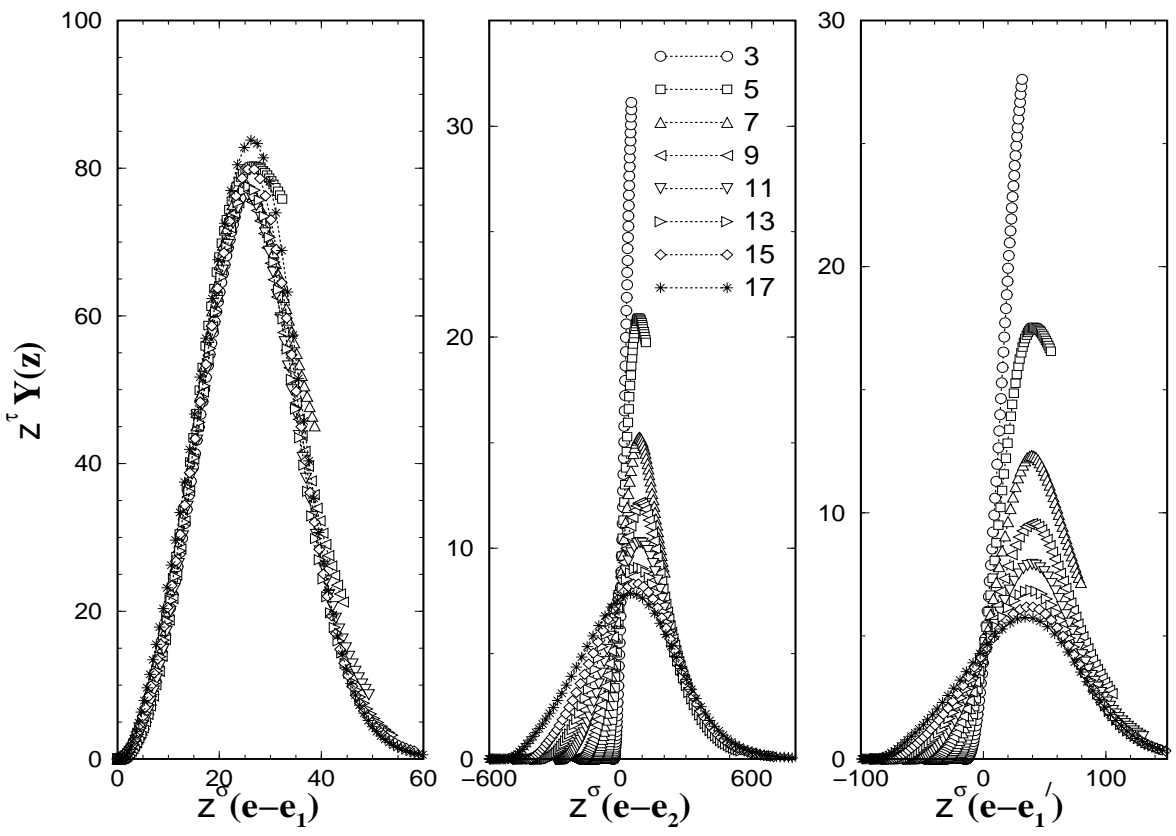

Fig.6: Similar to Fig.3 except that the thermodynamic model is used and scaling is tested around $e_{1}, e_{2}$ and $e_{1}$ '.

\section{IV. $8 \mathrm{GEV} / \mathrm{C} \pi^{-}$ON AU DATA}

It is of interest to check if the conclusions reached in the theoretical models are verified in experimental data 15 , 16 . Several non-trivial issues need to be clarified before this can be attempted. Because the power-law fit is not exact, the extraction of $\tau$ from data or theoretical calculation has some ambiguity. In previous sections we calculated $\tau$ by minimising the quantity defined as $\chi^{2}$ in eq.(2.1) at each $e$. But one could also minimise 


$$
\tilde{\chi}^{2} \equiv \sum\left(Y(z)-C z^{-\tau}\right)^{2} .
$$

If one is using experimental data, a more standard practice would be to minimise 17

$$
\hat{\chi}^{2} \equiv \sum \frac{\left(Y(z)-C z^{-\tau}\right)^{2}}{\sigma(z)^{2}},
$$

where often the $\sigma$ 's are statistical errors. The difference in the value of $\tau$ extracted by minimising Eq.(2.1) or Eq.(4.1) can be significant or small. In theoretically calculated values of $Y(z)$ the difference is small. But it is not so in the experimental values of $Y(z)$. For the experimental data of $8 \mathrm{GeV} / \mathrm{c} \pi^{-}$on gold, the results of using Eqs. (2.1), (4.1) and (4.2) are shown in Fig. 7. One can give crude arguments that minimising (2.1) rather than (4.1) means that $Y(z)$ 's of higher $z$ 's are preferentially fitted. In [18], Eq. (4.2) was chosen.

In order to compare the experimental results to the theoretical calculations we have taken the experimental yields

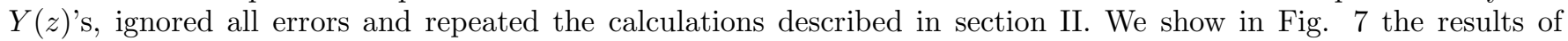
this analysis. Qualitatively, the results are similar to the theoretical results (Figs.1 and 4). In those two figures the minimum in $\tau$ occurs at very low excitation energy for the WC case. For the experimental data the minimum, if it exists, is also at a low value, below $1.5 \mathrm{MeV}$. More interesting is the right panel of Fig. 7 where we plot the experimental specific heat and find the maximum in specific heat coincides quite well with the minimum of $\chi^{2}$ (this is $\left.e_{1}^{\prime}\right)$. This agrement is also quite close to the theoretical predictions. The specific heat was extracted by differentiating with respect to $T$, the experimental caloric curve obtained for the same data set by A. Ruangma et al. [19].

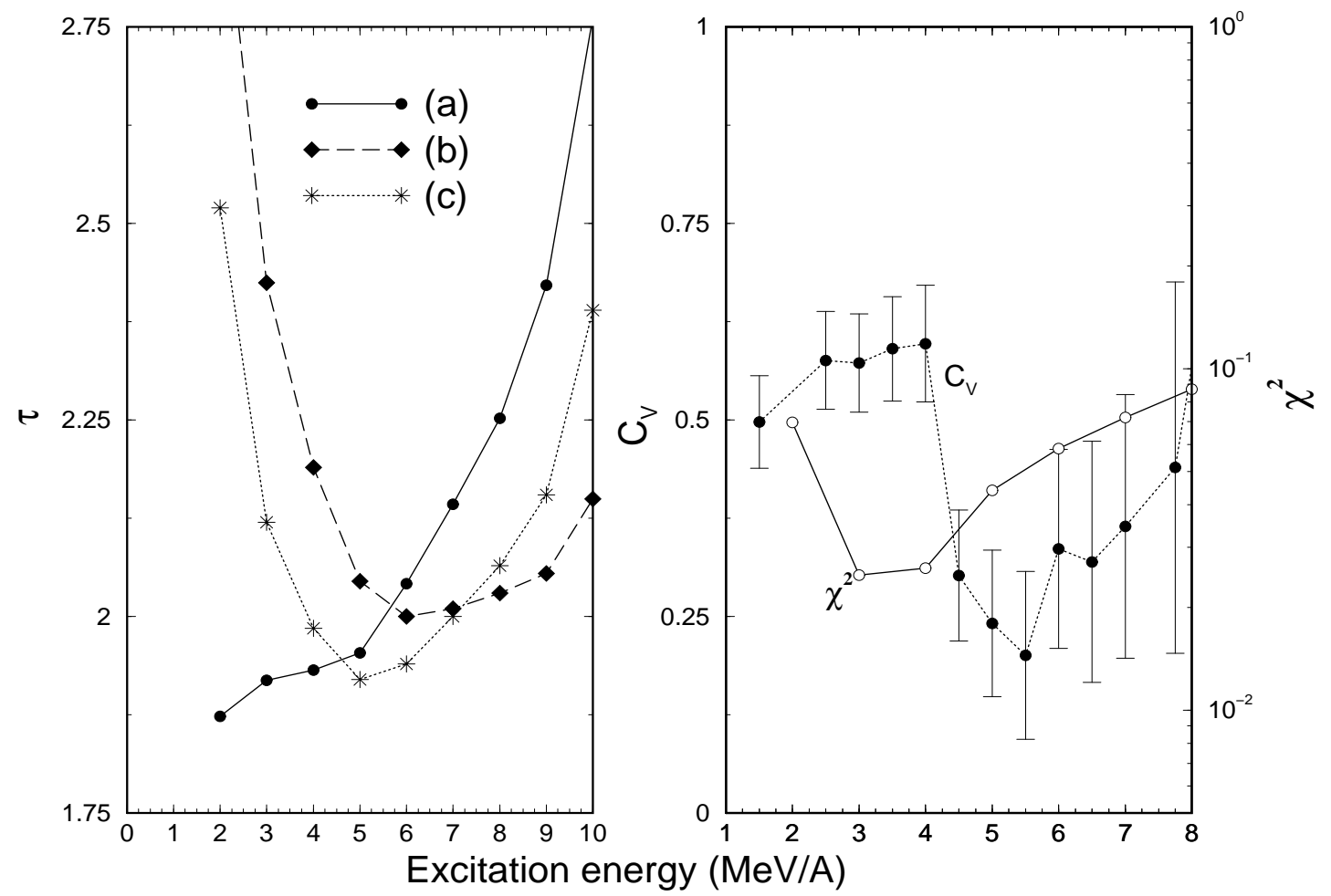

Fig.7: The left panel shows the values of $\tau$ extracted from the ISiS data using three different fomulae: (a) by minimising the right hand side of Eq.(2.1) at each e value, (b) by minimising the right hand side of Eq.(4.1) and (c) by minimising the right hand side of Eq.(4.2). In the right panel $C_{V}$ from data and $\chi^{2}$ (Eq.(2.1)) calculated from data are plotted. The minimum of $\chi^{2}$ and maximum of $C_{V}$ coincide within experimental uncertainty.

We also test the scaling around $e_{1}^{\prime}$ (Fig. 8) although some qualifying comments need to be made about this figure. In experiments, the source size as well as the charge of the thermal source, depend upon $e$. The scaling law, which spans $e$ values on either side of $e_{1}^{\prime}$, assumes constant source size as well constant charge. Thus the scaling law can not be directly tested without additional corrections renormalising the yields $Y(z)$ to compensate for changes in the source size and charge. This was not done here. (However the $\tau$ values and the values of $\chi^{2}$ should be insensitive to such changes although we do require that for a given $e$, the source size and charge remain unchanged. This last condition is approximately obeyed.)

We can summarise the results of the comparison with experimental data as follows. In the data the maximum of specific heat $\left(e_{2}\right)$ and the minimum of $\chi^{2}, e_{1}^{\prime}$ both are $e \approx 4 M e V$ with a value of $\tau$ about 2.1 and $\sigma$ about 0.53 . This 
is very close to to the results of Elliott et al. 20] for the same data set using Fisher's droplet model approach and Berkenbusch et al. 21] using a percolation model. For percolation, the excitation energy was defined as the critical excitation energy in the sense of a second order phase transition. The thermodynamic model, which has no adjustable parameters and only a first order phase transition, reproduces trends of the data very well although both $e_{2}$ and $\sigma$ are higher, $6 \mathrm{MeV}$ and 0.96 respectively.

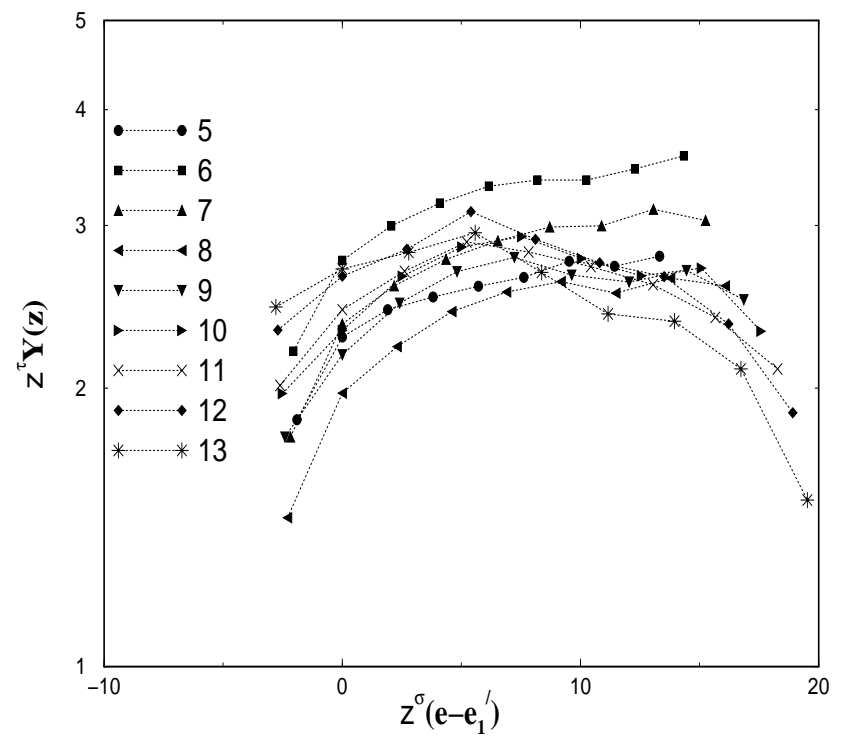

Fig.8: Scaling behavior associated with the ISiS data, around $e_{1}^{\prime}$.

Since source sizes change with excitation per particle, we tested the sensitivity of model calculations with regard to size using the thermodynamic model. Table II gives the results. Within the range of variation of relevance to the experimental data the changes are small, though not negligible.

\section{SUMMARY AND CONCLUSION}

We have discussed three characteristic excitation energies (equivalently temperatures): two of them have their origin in Eq.(1.2) ( $e_{1}=$ value of $e_{0}$ which gives best scaling and $e_{1}^{\prime}=$ value of $e_{0}$ where deviation from power-law is the least) and the third, $e_{2}$, is obtained from an extremum in specific heat. In an ideal situation, all three would have the same value. In theoretical models they are close if the Coulomb force is omitted. The Coulomb force makes a substantial splitting between $e_{1}$ and $e_{1}^{\prime}$, indicating the sensitivity of the extracted thermodynamic quantities to this interaction. We find that $e_{1}^{\prime}$ gives a better measure of the phase transition energy and it stays close to $e_{2}$. In the experimental data that we considered $e_{1}^{\prime}$ and $e_{2}$ follow this pattern.

The quantitative results are dependent upon freeze-out densities and the source sizes but not sensitively so.

Lastly, we have not discussed the order of phase transition but the model calculations in sections 2 and 3 imply a first order phase transition. In this interpretation, depending upon the excitation energy, most fragments are emitted while inside the co-existence region of the phase diagram (and possibly the spinodal region) and the extracted "critical" excitation energy indicates the boiling point. As pointed out earlier, the boiling point is at a similar excitation energy as the critical point found by Elliott et al. [20] from an analysis based on the Fisher droplet model and Berkenbusch et al. [21] based on percolation theory. Further discussions of critical phenomena [20,21] in disassembly of hot nuclei as opposed to first order phase transition in the disassembly can be found in 12,22,23

A first order phase transition is consistent with recent observations by the ISiS collaboration [16,24,25 of a strong increase in fragment production probability, a strong decrease in fragment emission time, and the onset of collective radial expansion above $4 \mathrm{~A} \mathrm{MeV}$ of excitation energy, which were interpreted as signatures for bulk emission. It is also consistent with the flattening of the caloric curve from which the heat capacity was extracted [19]. 


\section{ACKNOWLEDGMENT}

This work is supported in part by the Natural Sciences and Engineering Research Council of Canada and by le Fonds pour la Formation de Chercheurs et l'aide à la Recherche du Québec. Experiment E900 was supported by the US Department of Energy and the National Science Foundation.

[1] S. Das Gupta, A. Z. Mekjian and M. B. Tsang, Advances in Nuclear Physics, vol.26, 91 (2001).

[2] D. Stauffer and A. Aharony, Introduction to Percolation Theory (Taylor and Francis, London, 1992).

[3] J. Pochodzalla et al., Phys. Rev. Lett. 75, 1040 (1995).

[4] A Coniglio and W. Klein, J. of Phys. A13, 2775 (1980) 223(1981).

[5] J. Pan and S. Das Gupta, Phys. Rev C51,1384 (1995).

[6] S. Das Gupta and A. Z. Mekjian, Phys. Rev C57, 1361 (1998).

[7] P. Bhattacharyya, S. Das Gupta and A. Z. Mekjian, Phys. Rev C60, 54616 (1999).

[8] J. P. Bondorf, A. S. Botvina, A. S. Iljinov, I. N. Mishustin, and K. Sneppen, Phys. Rep. 257, 133 (1995).

[9] C. B. Das, S. Das Gupta and S. K. Samaddar, Phys. Rev. C63, 011602(R), (2001).

[10] S. K. Samaddar and S. Das Gupta, Phys. Rev C61, 034610 (2000).

[11] X. Campi and H. Krivine, Nucl. Phys. A620, 46 (1997).

[12] C. B. Das, S. Das Gupta and A. Majumder, Phys. Rev. C65, 034608 (2002).

[13] F. Gulminelli and Ph. Chomaz, Phys. Rev. Lett. 82, 1402 (1999).

[14] R. P. Scharenberg et al., Phys. Rev. C64, 054602 (2001).

[15] T. Lefort et al., Phys. Rev C64, 064603 (2001).

[16] L. Beaulieu et al., Phys. Rev. C64, 064604 (2001).

[17] W. H. Press, B. P. Flannery, S. A. Teukolsky and W. T. Vetterling, Numerical Recipes, (Cambridge University Press, New York, 1987) pp. 504 .

[18] L. Beaulieu et al., Phys. Lett. B463, 159 (1999).

[19] A. Ruangma et al., nucl-ex/010004, submitted to PRC.

[20] J. B. Elliott et al., Phys Rev. Lett 88, 042701 (2002)

[21] M. K. Berkenbusch et al., Phys. Rev. Lett. 88, 022701 (2002)

[22] J. Pan, S. Das Gupta and M. Grant, Phys. Rev. Lett. 801182 (1998)

[23] F. Gulminelli, Ph. Chomaz, M. Bruno and M. D'Agostino, Phys. Rev.C65, R 051601 (2002)

[24] L. Beaulieu et al., Phys. Rev. Lett 84, 5971 (2000)

[25] T. Lefort et al., Phys. Rev C62, R031604 (2000)

TABLE I. The values of the parameters $e_{1}, e_{2}$ and $e_{1}^{\prime}$, corressponding $T_{1}, T_{2}$ and $T_{1}^{\prime}$; and $\tau^{\prime}$ s at these $e_{1}, e_{2}, e_{1}^{\prime}$ at $e_{1}$ as obtained in LGM and thermodynamic models, for freeze-out density of $0.27 \rho_{0}$. Values are shown for calculations with (WC) and without (NC) Coulomb interactions.

\begin{tabular}{ccccc}
\hline \hline$\overline{\text { Parameters }}$ & LGM (NC) & LGM (WC) & THDM (NC) & THDM (WC) \\
\hline$e_{1}$ & 11.66 & 3.60 & 6.77 & 0.75 \\
$T_{1}$ & 4.46 & 1.95 & 7.53 & 2.94 \\
$\tau\left(e_{1}\right)$ & 1.964 & 1.771 & 2.77 & 2.36 \\
$e_{2}$ & 12.61 & 8.38 & 8.01 & 6.03 \\
$T_{2}$ & 4.62 & 3.38 & 7.715 & 6.445 \\
$\tau\left(e_{2}\right)$ & 1.824 & 1.251 & 2.44 & 1.55 \\
$e_{1}^{\prime}$ & 13.88 & 10.5 & 7.54 & 4.94 \\
$T_{1}^{\prime}$ & 4.88 & 3.82 & 7.65 & 6.05 \\
$\tau\left(e_{1}^{\prime}\right)$ & 2.544 & 1.851 & 2.53 & 1.42 \\
\hline \hline
\end{tabular}


TABLE II. The values of the parameters for two different sizes of the fragmenting source as obtained in the thermodynamic model, for a freeze-out density of $0.27 \rho_{0}$.

\begin{tabular}{ccc}
\hline \hline Parameters & $\mathrm{N}=118$ & $\mathrm{~N}=101$ \\
$\mathrm{Z}=68$ & $\mathrm{Z}=79$ & 1.01 \\
\hline$e_{1}$ & 0.75 & 3.46 \\
$T_{1}$ & 2.94 & 2.35 \\
$\tau\left(e_{1}\right)$ & 2.36 & 5.90 \\
$e_{2}$ & 6.03 & 6.40 \\
$T_{2}$ & 6.445 & 1.49 \\
$\tau\left(e_{2}\right)$ & 1.55 & 4.91 \\
$e_{1}^{\prime}$ & 4.94 & 6.05 \\
$T_{1}^{\prime}$ & 6.05 & 1.41 \\
$\tau\left(e_{1}^{\prime}\right)$ & 1.42 & \\
\hline \hline
\end{tabular}

